\title{
AES Cryptograph for Secure Cloud Storage
}

\author{
B. Mahesh, K. Pavan Kumar, M. Jahir Pasha
}

\begin{abstract}
In cloud computing disseminated assets are shared by means of system in open condition. Subsequently client can without much of a stretch access their information from anyplace. Simultaneously exist protection and security problems because of numerous causes. Initial one is emotional improvement in system advances. Another is expanded interest for computing assets, which make numerous associations to reappropriate their information stockpiling. So there is a requirement for secure cloud stockpiling administration in open cloud condition where the supplier isn't a confided in one. Our research tends to various information security and protection assurance problems in a cloud computing condition and suggests a technique for giving diverse security administrations like validation, approval and classification alongside checking in postponement. 128 piece Advanced Cryptograph Standard (AES) is utilized to increment information security and classification. In this supported methodology information is encoded utilizing AES and afterward transferred on a cloud. The supported model uses Short Message Service (SMS) ready instrument with keeping away from unapproved access to client information.
\end{abstract}

Keywords: Network, Authorization, Confidentiality, Security, authorized searchable cryptograph

\section{INTRODUCTION}

The cloud computing model incorporates a few innovative progressions, for example, virtualization, Internet administrations, and SLA the executives for big business software. Because of fast advancement in advances increasingly more specialist co-ops and clients using cloud condition. Today different government organizations and business endeavor frameworks are utilizing distinctive cloud administrations to give arrange network and high administration accessibility to the end clients. Cloud suppliers provide their administrations in three basic models: IaaS, PaaS and SaaS. Despite the fact that cloud computing has numerous preferences when contrasted and the conventional information stockpiling instruments; security cover is a hindrance for picking cloud computing from the buyers view point. The analysts are completed a few examinations identified with security problems in cloud. Cloud foundation is fundamentally accessible out in the open and restricted mode. Private cloud is committed to a solitary client or association. The facilitated administrations are offered to restricted number of people groups, this limits the security cover. In open cloud, the foundation is possessed and managed by cloud supplier itself. Henceforth security and secrecy of information is a significant concern. As the quantity of cloud clients builds step by step, the QoS

B. Mahesh, Department of CSE, Dr.K.V.Subba Reddy College of Engineering for Women, Kurnool, A.P, India (Email:mahesh.bhasutkar@gmail.com)

K. Pavan Kumar, Department of CSE, Dr.K.V.Subba Reddy College of Engineering for Women, Kurnool, A.P, India. (Email:kpavankumar502@gmail.com)

M. Jahir Pasha, Department of CSE, Dr.K.V.Subba Reddy College of Engineering for Women, Kurnool, A.P, India. (Email: jahir4444@gmail.com)
Revised Manuscript Received on 14 August, 2019.

the executives is another significant issue. QoS the board in cloud computing condition alludes to the exercises in QoS determination, for example, assessment, expectation, collection and standard of assets to meet start to finish client and application necessities. With the development in innovations an enormous number of associations like Amazon, FlipKart,GitHub and so on., have just put resources into cloud computing. Huge number clients share colossal measure of information at high speeds from geologically scattered areas. Be that as it may, in genuine cloud computing condition existing arrangements are inclined to disappointment and security bargain in numerous regions: computing execution, cloud unwavering quality and data security. Present methodologies are not adequate to guarantee information security for end clients. The supported methodology give an unmistakable and compact perspective on deferral inside genuine cloud computing situations and illuminate cloud clients about unapproved access to their information through a SMS ready framework. This paper talks about various research works accomplished for the board and observing of various QoS parameters in cloud. And furthermore gives a unique perspective on cryptograph systems AES, DES and RSA.

\section{SYSTEM MODEL}

Prior to talking around the supported framework in detail, we need to think about security problems in cloud condition and significance of AES among other cryptograph calculations.

\section{A. Cloud Security Issues}

1) Cloud Security: Cloud computing security (now and then alluded to just as "cloud security") is a developing subdomain of System security, arrange security, and, more comprehensively, data security [5]. It alludes to a wide arrangement of arrangements, innovations, and controls conveyed to ensure information and the related foundation of cloud computing.

2) Security Problems related with the Cloud: Few security problems exist within cloud computing. Determination of cloud sellers, clients ought to get some information about seven issues: Privileged client get to, administrative consistence, information area, information isolation, recuperation, analytical help also, long haul suitability [6]. The CSA has recognized security problems in various cloud spaces and further more give security directions [8]. An overview of security problems in cloud in administration conveyance models and given a nitty gritty examination every security issue in [8]. Server farm skills is a main online wellspring of every day reports, they broke down information focus security problems. They reports Security Breaches, Data Misfortune, Outages happened in cloud [2].

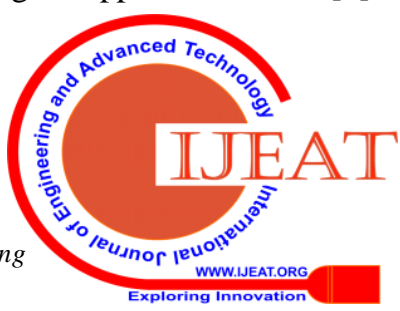




\section{B. Cryptograph calculations}

The cryptograph calculations predominantly classified into two: Symmetric and Asymmetric key cryptographs. In symmetric key cryptograph solitary mystery key is utilized for both cryptograph and decoding. In hilter kilter key, cryptograph is executed utilizing open key and decoding utilizing mystery key. AES and Data Cryptograph Standard (DES) are two symmetric key cryptograph techniques. RSA is model for deviated key cryptograph.

1) DES:The calculation likewise alluded as Data Cryptograph Calculation (DEA). For DES information are encoded in 64-bits squares utilizing 56-bit key. This calculation change 64-bit into a 64-bit yield through a progression of steps. Similar advances and key is utilized for unscrambling moreover. In the underlying advance the 64bit plain content goes through an underlying stage. Next stage comprise of 16 rounds of both change and interchange capacities. Last round comprise of 64-bit yield: the left and right $50 \%$ of the yield is swapped, this will produce the preyield. In the last advance invert of introductory change is connected to the pre-output, which produces 64-bit figure content. DES at long last and absolutely demonstrated unreliable by the EFF foundation, they reported that the DES cryptograph is conceivable to broken by utilizing specific reason DES saltine machine that was worked for $\$ 270,000$. As indicated by them under three days required for breaking DES cryptograph. Luckily there are various options to DES like AES, Triple DES. DES is progressively helpless against beast power assault in light of its short key span (56 bit).

2) AES: AES is symmetric square figure that is supported to replaces DES. The figure takes plaintext of size 18-bit. The key span can be 128-bits,192-bits,256-bits. The calculation alluded to as AES-128, AES-192 and AES-256 relying upon key span. The figure comprises of $\mathrm{N}$ rounds relies upon key span: 10 rounds for a 128 piece key, 12 rounds for a 192 piece key and 14 rounds for 256 bit key. First N-1 rounds comprise of 4 change capacities One stage and three substitutions . Last round of both cryptograph and unscrambling comprise of just 3 phases. Substitute bytes use $\mathrm{S}$ box for byte by byte substitution. MixColumns utilizes number-crunching over GF (28) Add RoundKey is straightforward piece shrewd XOR of present square with a part of extended key.

3) RSA: In RSA the plaintext and figure content are whole numbers among zero and $n-1$. The run of the mill size of $n$ is 1024 piece. Cryptograph and unscrambling are of the accompanying structure for a plain content square A and figure content square $\mathrm{C}$.

$$
\begin{aligned}
& C=A^{x} \bmod b \quad-(1) \\
& A=C^{y} \bmod b=A^{x y} \bmod b
\end{aligned}
$$

Here " $x$ " and " $y$ " are public and private keys. This algorithm has been used in various applications like ecommerce trade which ensures probity, privacy, validation and non repudiation.

\section{Cause of AES Cryptograph}

1) Comparative examination: Table I demonstrates the similar examination of cryptograph calculations - DES, AES and RSA based on key span, figure type, square size, security, effortlessness in equipment and programming usage, cryptograph/decoding speed and so forth. A near report between various cryptograph strategies AES, DES and RSA dependent on animated time for cryptograph and unscrambling has processed in [3]. Distinctive size content records are utilized as contribution for assess the cryptograph and decoding time. In view of their tests they finished up that AES calculation expends least cryptograph and RSA expend longest cryptograph time. In view of their outcomes they come to in a resolution that AES calculation is greatly improved than DES and RSA calculation. From table I demonstrate that RSA is least secure and AES is most secure and quicker one. Presently a days a significant issue looked by all consortium and providers is that quickest and secure conveyance of administrations to the clients. Security of any framework likewise relies upon client fulfillment stage. Thus the supported framework gave security to client information through cryptograph before transferring on the cloud. AES calculation is utilized for information cryptograph and decoding, since it is quicker and secure than different calculations.

Table I. Comparison between aes, des and rsa

\begin{tabular}{|l|l|l|l|}
\hline Attributes & DES & AES & RSA \\
\hline $\begin{array}{l}\text { Come into } \\
\text { existence }\end{array}$ & 1977 & 2000 & 1977 \\
\hline KeySpan & 56 -bits & $\begin{array}{l}128- \\
\text { bits,192- } \\
\text { bits,256- } \\
\text { bits }\end{array}$ & $>1024$-bits \\
\hline CipherType & $\begin{array}{l}\text { Symmetric } \\
\text { block } \\
\text { cipher }\end{array}$ & $\begin{array}{l}\text { Symmetric } \\
\text { block } \\
\text { cipher }\end{array}$ & $\begin{array}{l}\text { Asymmetric } \\
\text { block cipher }\end{array}$ \\
\hline BlockSize & 64-bits & 128-bits & $\begin{array}{l}\text { Minimum } \\
512-\text { bits }\end{array}$ \\
\hline Security & $\begin{array}{l}\text { Not secure } \\
\text { enough }\end{array}$ & $\begin{array}{l}\text { Excellent } \\
\text { secured }\end{array}$ & Least secure \\
\hline $\begin{array}{l}\text { Hardware \& } \\
\text { Software } \\
\text { Implementation }\end{array}$ & $\begin{array}{l}\text { Better in } \\
\text { hardware } \\
\text { than } \\
\text { software }\end{array}$ & $\begin{array}{l}\text { Better in } \\
\text { both }\end{array}$ & $\begin{array}{l}\text { Not } \\
\text { efficient }\end{array}$ \\
\hline $\begin{array}{l}\text { Cryptograph } \\
\text { and } \\
\text { Decryption } \\
\text { Slower }\end{array}$ & Moderate & Faster & Slower \\
\hline
\end{tabular}

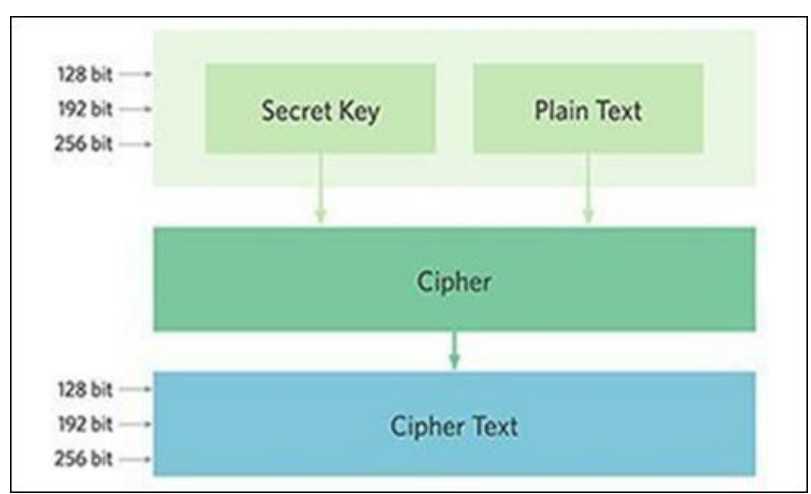

Fig. 1. Data Cryptograph with AES 


\section{Issue Statement}

The data stockpiling in cloud is like data put away in other capacity gadgets yet in isolated areas. In cloud the client can get to their data at whenever from anyplace. Three parts of data security need to think about when utilizing cloud administrations: classification, integrity and accessibility. Open cloud framework give adaptable and on interest information stockpiling. This keeps away from the weight of creation and upkeep of private framework for information stockpiling. The clients get a few advantages i.e unwavering quality, accessibility with least expense and exertion. In any case, there is some security and protection dangers. One significant issue on this is secrecy of client data. One normal answer for keep up data classification is cryptograph. To guarantee adequacy of cryptograph there must utilize effective cryptograph calculation. In cloud computing condition where a lot of information transmission, capacity and taking care of happen, consequently likewise need to consider handling speed just as computational proficiency of cryptograph calculation. For this situation symmetric cryptograph calculation is more appropriate than hilter kilter cryptograph calculation.

To address the above issues and increment the quantity of clients, this paper suggests another methodology dependent on AES cryptograph system. Fig. 1 demonstrates the security model of supported approach. The supported methodology will guarantee the following highlights:

- $\quad$ Privacy - The cloud stockpiling supplier don't know any data about client information.

- Integrity - Any unapproved access to client information is dealt with by SMS ready component.

Alongside this, the current highlights of cloud was moreover upheld, for example

- Availability - Customer can get to their information from any machine whenever utilizing their mystery file_id.

- Data sharing - Customers can give access to their data with confided in gatherings.

The greater part of the past examinations are not done analyses in genuine cloud condition. So a progressively solid and secure successful framework which is tried in genuine condition is required for secure information stockpiling.

\section{E. Framework Module}

The supported framework having three parts: cloud controller, customer and various hubs. Postponement estimation was processed dependent on the solicitation and reaction time during record transfer. Fig. 2 demonstrates the in general framework engineering.

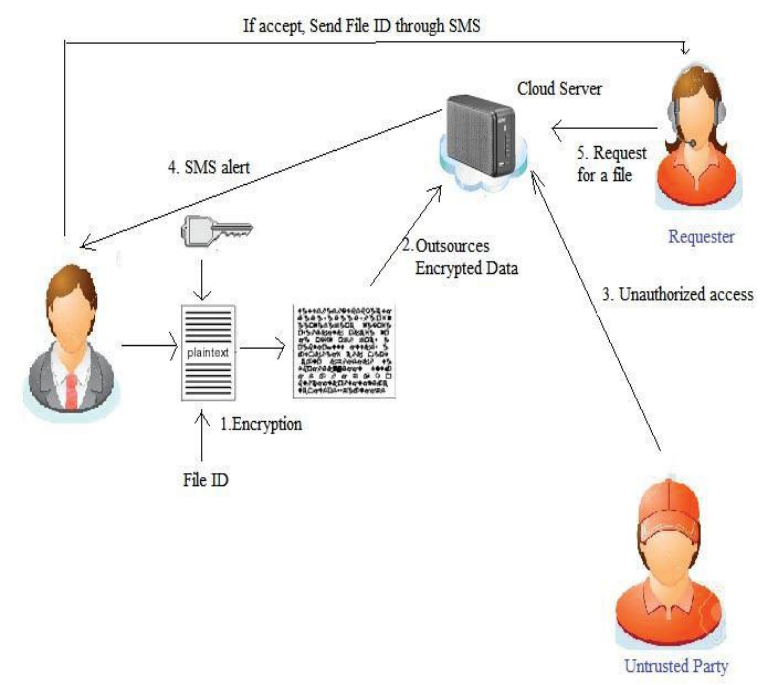

Fig 2. System Architecture

\section{RESULT}

The supported framework based on a model of an public record preparing application. The application was facilitated in an public cloud database given by the cloud supplier. The supplier is a US based cloud specialist co-op. A Supplier record was made for running the application. In this model one framework is go about as the controller. Anyone can get to the application from anyplace whenever over web. Various web programming languages are the programming dialects utilized for making this application. Graphical Uuser Interface was made with Hyper Text Markup Language. Fig. 2 demonstrates the general framework engineering and exercises in supported model. One significant utilization of this framework is secure sharing of classified information like therapeutic record, individual data, money related data and so forth. Assume a client needs to get to our application for transferring their secret information, she/he should register with their legitimate emailid and versatile number with our framework. The username and secret word for their record is client characterized and not framework characterized. After fruitful enrollment they can login as a client. At that point client can transfer the secret record through document transfer module. Before transferring document to cloud, the client gets a canvas for scrambling their document as individual squares. At that point click the spare catch in the wake of setting a mystery file ID for future getting to and sharing. The record will transfer to the Supplier server database. On account of medicinal record, client can impart record to their primary care physician at whenever from anyplace, there is no requirement for keeping their records with them continuously as printed version or delicate duplicate. Just need is to recall their mystery record ID. The document ID might be numbers, alphabetic and number characters or uncommon characters as client wish or they can utilize a mix of these as document ID. There is no confinement for length of file_id. The client can view time taken for transferring their record. 


\section{A. Security}

The supported model utilized 128 piece AES cryptograph. The cryptograph comprises of 10 rounds for 128-piece keys. In this model, the document was part into various squares relying upon record size. At that point individual squares are encoded independently. After square savvy cryptograph each square transferred to cloud at various areas with file_id and block_id. In the event that anyone like cloud supplier, attempt to get to a record straightforwardly from the server, they can't get entire information, since it put away at various areas and furthermore in encoded structure. Thus the individual who knows mystery file_id can recovers information. The supported framework gives a web based altering office, for example client can alter their information and after that transferred on to cloud without downloading to their framework. Just the genuine client can utilize this office while others can as it were see information. Fig. 3 demonstrates the diagrammatic portrayal of record cryptograph .

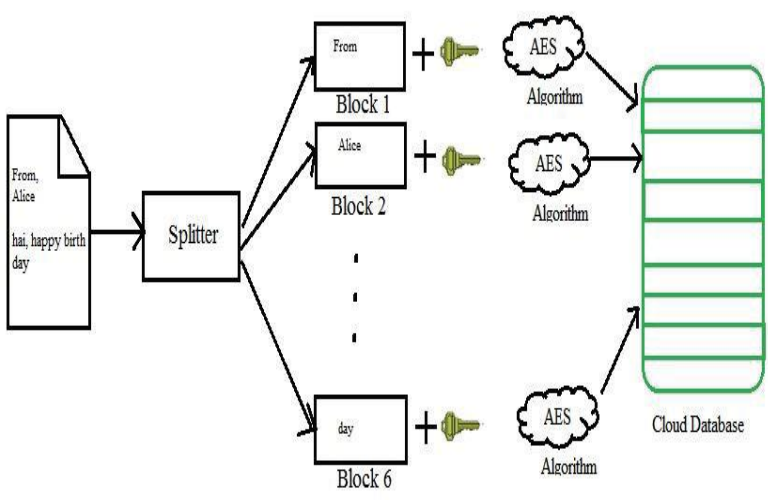

Fig 3. Cryptograph

\section{B. Deferral}

Deferral is the major factor considered while assessing the QoS in each framework. Postponement relies upon different components, including mis-arrangement of programming stack, blocked ports in system, what's more, information preparing delays. In this framework record transferring and downloading postponement was observed at various time interim. Fig 4 shows watched postponement and determined defer when transferring documents with various sizes.

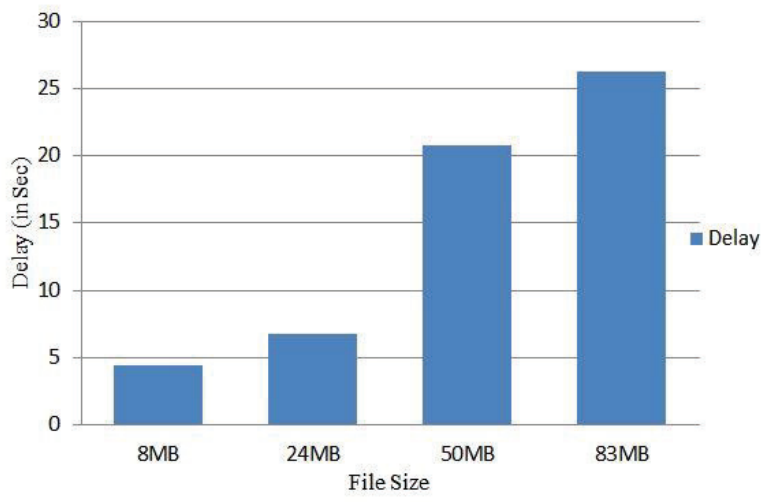

Fig 4.Delay occur when uploading files with different sizes.

\section{CONCLUSION AND FUTURE WORK}

It is concentrated existing security issues in cloud computing condition and supported another strategy for verifying cloud information in genuine condition. 128 piece AES cryptograph is utilized for giving secrecy, realness what's more, get to control. At that point execution of supported approach was investigated dependent on deferral. From this investigation we watched that there is uncommon increment in deferral with increment in record size. Future work suggests another strategy for astute information capacity in which the capacity hubs are evaluated based on the past assault history.

\section{REFERENCES}

1. "Distributed Shared Memory Programming in the Cloud", Ahmad Anbar, Vikram K. Narayana, Tarek ElGhazawi, Proceedings of the 2012 12th IEEE/ACM International Symposium on Cluster, Cloud and Grid Computing (ccgrid 2012), p.707-708, May 13-16, 2012

2. http://www.datacenterknowledge.com/archives/2015/03/ 16/securitybreaches-data-loss-outages-the-bad-side-ofcloud/

3. "A Study of Cryptograph Algorithms AES, DES and RSA for Security", Prerna Mahajan, Abhishek Sachdeva, Global Journal of Computer Science and Technology Network, Web \& Security, Vol.13, Iss. 15, Vol. 1, 2013.

4. Trust and Reputation Calculation and Management", Dr.B.Mahesh, IJETAE, Vol 7, Iss, 11, 2017, pp. 93-96, https://ijetae.com/files/Volume7Issue11/IJETAE_1117_1 6.pdf

5. "Security Guidance for Critical Areas of Focus in Cloud Computing V2.0" http://www.cloudsecurityalliance.org/ guidance/csaguide.v2.1.pdf, retrieved on 19th November 2015.

6. "Research on cloud computing security problem and strategy", Wentao Liu, IEEE 2nd International Conference on Consumer Electronics, Communications and Networks (CECNet), 2012, pp. 1216-1219.

7. "Gartner: Seven cloud-computing security risks". InfoWorld.

2008-07-02

http://www.infoworld.com/d/security-central/gartnerseven-cloudcomputing-security-risks-853, retrieved on 6th March 2016.

8. "The Significance of Data Security in Cloud: A Survey on Challenges And Solutions on Data Security", Ashalatha R, Vaidehi M ,International Journal of Internet Computing, Vol, 1, Iss. 3, 2012, pp.15-18. 